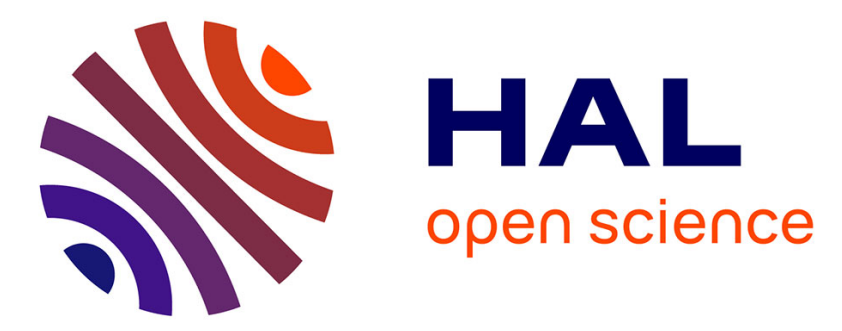

\title{
Size effects of the magnetic anisotropy of fcc cobalt nanoparticles embedded in copper
}

Matthias Hillenkamp, Simón Oyarzún, Nicolas Troc, Julien Ramade, Alexandre Tamion, Florent Tournus, Veronique Dupuis, Varlei Rodrigues

\section{To cite this version:}

Matthias Hillenkamp, Simón Oyarzún, Nicolas Troc, Julien Ramade, Alexandre Tamion, et al.. Size effects of the magnetic anisotropy of fcc cobalt nanoparticles embedded in copper. The European Physical Journal D : Atomic, molecular, optical and plasma physics, 2017, 71 (12), pp.330. 10.1140/epjd/e2017-80299-x . hal-02004432

\section{HAL Id: hal-02004432 \\ https://hal.science/hal-02004432}

Submitted on 14 Feb 2019

HAL is a multi-disciplinary open access archive for the deposit and dissemination of scientific research documents, whether they are published or not. The documents may come from teaching and research institutions in France or abroad, or from public or private research centers.
L'archive ouverte pluridisciplinaire HAL, est destinée au dépôt et à la diffusion de documents scientifiques de niveau recherche, publiés ou non, émanant des établissements d'enseignement et de recherche français ou étrangers, des laboratoires publics ou privés. 


\title{
Size effects of the magnetic anisotropy of fcc cobalt nanoparticles embedded in copper
}

\author{
Matthias Hillenkamp · Simón Oyarzún · \\ Nicolas Troc • Julien Ramade . \\ Alexandre Tamion - Florent Tournus . \\ Véronique Dupuis · Varlei Rodrigues
}

Received: date / Accepted: date

\begin{abstract}
Cobalt nanoparticles embedded in copper matrices show strong size effects in the magnetic anisotropy with a non-monotonous dependence on the particle diameter. In this article we discuss quantitative values of the magnetic anisotropy in the frame of two models: in small clusters the surface anisotropy contribution dominates whereas larger particles ( $>3 \mathrm{~nm}$ diameter) have an elliptic shape leading to increased shape anisotropy. The crystalline structure of the particles is shown to be face-centered cubic, justifying that the magneto-crystalline anisotropy can be neglected.
\end{abstract}

Keywords nanoparticles $\cdot$ cluster $\cdot$ size effects $\cdot$ nanomagnetism $\cdot$ magnetic anisotropy

PACS 75.75.+a, 36.40.Cg, 81.07.-b

\section{Introduction}

Cluster-assembled metallic nanostructures, i.e. solid thin films with embedded nanoparticles are widely used to study specific physical effects arising due to the reduction of matter investigated to the nanoscale. These comprise,

M. Hillenkamp · S. Oyarzún · N. Troc $\cdot$ J. Ramade $\cdot$ A. Tamion $\cdot$ F. Tournus · V. Dupuis Univ. Lyon, Université Claude Bernard Lyon 1, CNRS, Institut Lumière Matière, F-69622, Villeurbanne, France

Tel.: +33 4.72.43.11.21

Fax: +33 4.72.43.15.07

E-mail: matthias.hillenkamp@univ-lyon1.fr

Present address: of S. Oyarzún

Departamento de Física, Universidad de Santiago de Chile. Avenida Ecuador 3493, Estación Central, Santiago, Chile

M. Hillenkamp · V. Rodrigues

Univ. Estadual Campinas Unicamp, Inst. Fis. Gleb Wataghin, Rua Sergio Buarque de Holanda 777, BR-13083859 Campinas, SP, Brazil 
amongst others, size effects, where, on the one hand, the reduced dimensionality can induce new crystalline phases such as fivefold symmetric icosahedral structures [1] and, on the other hand, the electronic confinement can lead to effects such as the localized surface plasmon resonance [2] or superparamagnetism [3]. We have demonstrated in a recent publication [4] that the advanced treatment of magnetic data from cobalt clusters embedded in copper matrices allows the highly accurate characterization and determination of properties such as the magnetic particle diameter distribution as well as the effective magnetic anisotropy constant, its dispersion about a mean value and a possible biaxial component. We have quantified size-dependent variations in the effective magnetic anisotropy constant of a factor of two in the range between 1.9 and $5.5 \mathrm{~nm}$. Here we describe in more detail the sample fabrication, support our interpretations with additional magnetic data and present structural characterization to support our magnetic findings and interpretations.

\section{Experimental: Sample Fabrication}

Cluster-assembled nanostructures are prepared by embedding pre-formed pure cobalt nanoparticles in co-evaporated copper matrices [5] at dilutions of $<1$ at.\% in order to avoid inter-particle interactions [6,7]. The $\mathrm{Co}_{n}$ cluster ions (n: number of atoms per cluster) are fabricated in a home-built magnetron sputter/aggregation source and guided through several differential pumping stages using different ion optical components.

Cobalt atoms are sputtered into the gas phase in a mixed argon/helium atmosphere of typically $\sim 0.1$ mbar and guided in a viscous flow towards the tube exit which is an adjustable iris. During this time metal atoms condense into clusters and as a rule of thumb the mean particle size is determined by the time available for metal-metal collisions before being swept out of the aggregation tube. The aggregation tube is kept at a positive potential with respect to ground of typically $+100-200 \mathrm{~V}$, thereby defining the final kinetic energy of the particles. The first potential difference, between the source tube and a floating skimmer with $6 \mathrm{~mm}$ opening, has to be small in order to avoid acceleration in this high pressure region, leading to heating and fragmentation of the particles. Larger nanoparticles ( $>\sim 3 \mathrm{~nm}$ diameter) have, due to the high kinetic energy obtained in the gas flow out of the aggregation tube and into vacuum, a comparably small angular dispersion. They can easily be transferred through the differential pumping stages via gentle electrostatic acceleration and focusing with an Einzel lens. Smaller clusters leave the cluster source into a bigger solid angle and their efficient transfer demands advanced guidance. In this case we use a radiofrequency octopolar ion guide attached to the skimmer in order to transfer them through the remaining high pressure region, thereby increasing the cluster current by an order of magnitude. Although many of the different source parameters show certain tendencies (e.g. bigger distance between discharge and aggregation tube exit makes bigger clusters, opening the iris at the aggregation tube exit reduces the pressure and makes them 


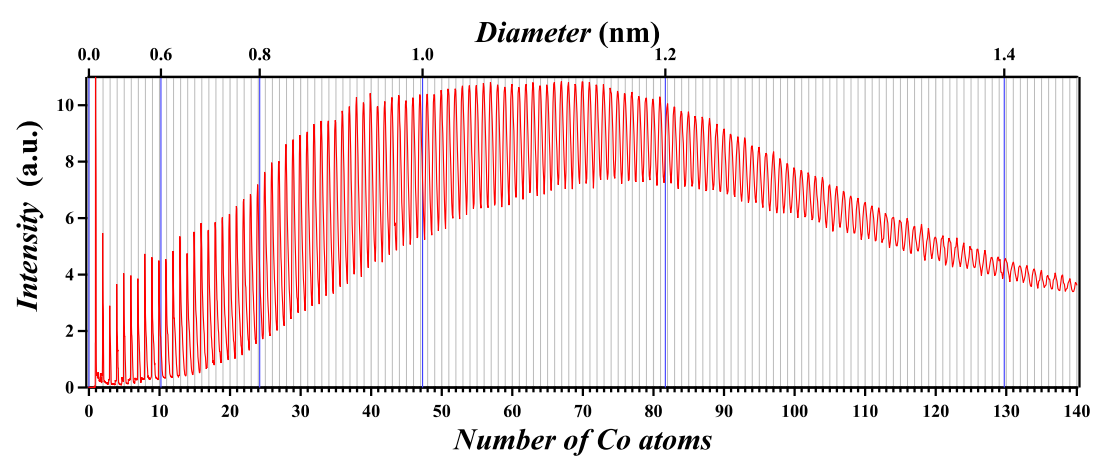

Fig. 1 (color online) Calibrated mass spectrum for cobalt clusters. Individual sizes are distinguished up to above 140 atoms/cluster.

smaller, ...) we noted that namely the electric potentials used to guide the beam through the following high pressure region crucially influence the mean transmitted size, its dispersion as well as the cluster ion beam intensity.

The cluster ion beam is then deviated in an electrostatic bender towards either a time-of-flight mass spectrometer (TOF-MS) for rapid in-situ characterization or towards the deposition chamber containing a second quadrupolar mass filter (QMS, used here as ion guide) as well as the sample holder with detectors. The silicon substrate is mounted at $45^{\circ}$ with respect to the cluster beam so as to allow for the simultaneous deposition of the copper matrix. The first step in the sample fabrication is the optimization of the cluster beam with TOF-MS. Once the mean particle size is adjusted, the intensity is optimized using the ion optical components. Typical obtained cluster ion currents on the sample holder in this work were in the range 60-240 pA on a detector surface of $3 \mathrm{~mm}^{2}$, which corresponds to particle fluxes of the order of $100 \mathrm{~s}^{-1} \mu \mathrm{m}^{-2}$. In order to calibrate the nanoparticle size, we record TOF mass spectra for smaller sizes under otherwise identical conditions. An example mass spectrum is shown in Fig. 1, displaying resolution up to $\mathrm{n}>150$ atoms/cluster. We have cross-checked this calibration method using Transmission Electron Microscopy (TEM). Note however that while the mean size of the nanoparticles actually deposited is very well reproduced in TOF-MS, the size dispersion can vary significantly. We attribute this to experimental conditions depending transmission of the QMS and consequently focus in this work on the comparison between magnetic and geometric size dispersions as derived from TEM, where available.

\section{Experimental Results}

\subsection{Structural characterization}

One important question is that of the crystalline structure of the embedded cobalt clusters. We have used electron microscopy in order to determine the 


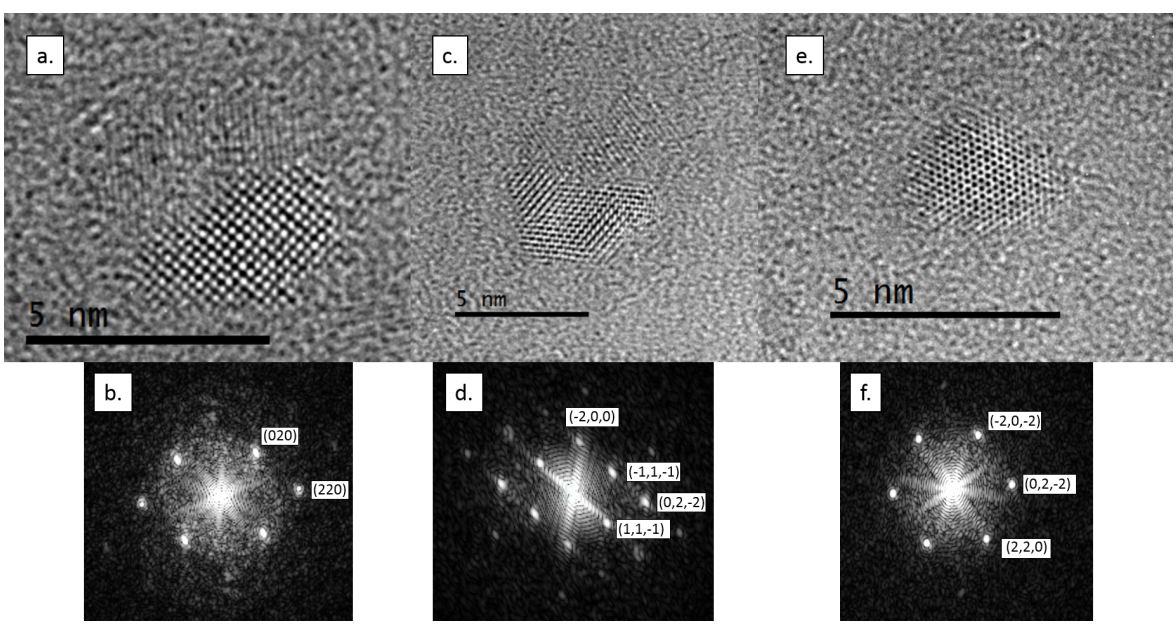

Fig. 2 HR-TEM images and the respective Fourier transform patterns of cobalt nanoparticles embedded in amorphous carbon. The particle diameters (mean of the two half axes) and orientations are, respectively: a, b: $4.6 \mathrm{~nm}[0,0,1]$; c, d: $5.0 \mathrm{~nm}[0,1,1]$ and e, f: $2.8 \mathrm{~nm}$ $[-1,1,1]$.

crystallographic structure of our nanoparticles. High Resolution TEM (HRTEM) has been performed using a FEI Titan environmental TEM at the Lyon Center for Microscopy (CLYM). Here the nanoparticles were encapsulated in a thin amorphous carbon layer. Amorphous carbon is much softer than cobalt, we consequently do not expect it to impose a specific crystalline structure onto the nanoparticles.

We have in the past verified for several matrix materials (including copper and carbon) the coherence between the derived size distributions [8] and have furthermore evidenced that magnetically dead metastable carbides at the interface do not alter the geometric size and can be removed by heat treatment [9]. All investigated particles with suitable orientation to allow indexing display fcc structure (sometimes polycrystalline), examples are displayed in figure 2. Note that some bigger particles, e.g. the one in figure 2a are twinned. However, no indication for multiply twinned (such as decahedral or icosahedral) or for hcp structures was observed.

\subsection{Magnetometry}

We have prepared samples with mean sizes ranging from 1.9 to $5.5 \mathrm{~nm}$ in diameter and measured their magnetic response as a function of applied field and temperature. The experimental curves were fitted using the extended "triple fit" procedure $[10,11]$. We notably fit the high temperature $m(H, T)$ curve where all nanoparticles are in the superparamagnetic state together with the zero-field cooled and field-cooled (ZFC/FC) susceptibility curves at small applied field $(5 \mathrm{mT})$. A high level of confidence is obtained through the simulta- 


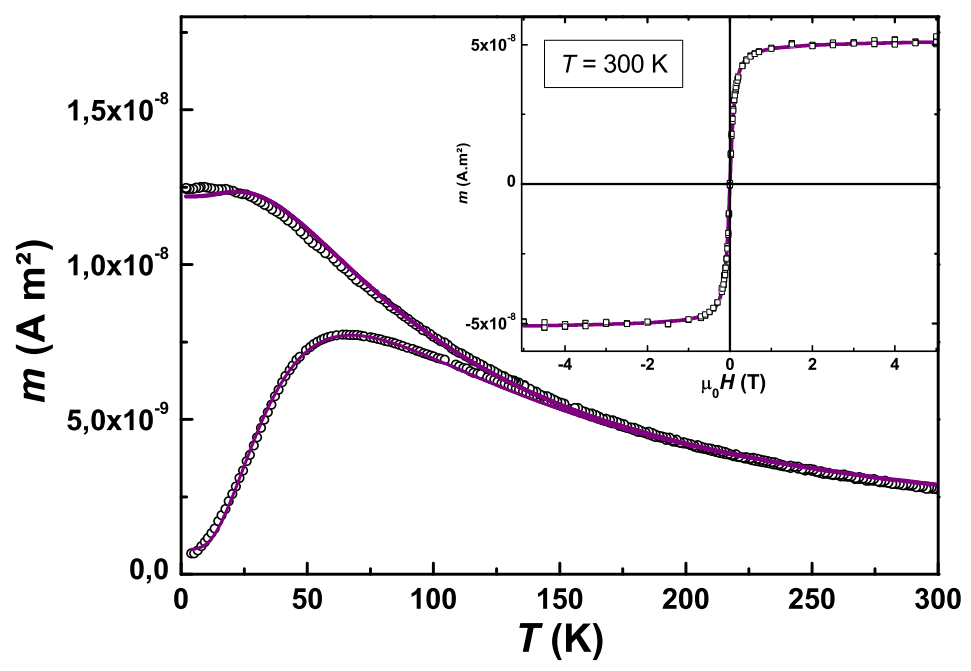

Fig. 3 (color online) Magnetic characterization of the $4.7 \mathrm{~nm}$ sample. Shown are the experimental $\mathrm{ZFC} / \mathrm{FC}$ curves at $5 \mathrm{mT}$ and the $m(H, T)$ magnetization curve at $T=300 \mathrm{~K}$ (points) together with the fits (solid lines).

neous and consistent fitting of several entire curves with only a small number of parameters: the number of particles in the sample (reflecting only the amplitude of the signal), the median magnetic diameter $D_{m a g}$ and its dispersion $w_{\text {mag }}$ in a log-normal description, the effective anisotropy constant $K_{\text {eff }}$ and a Gaussian anisotropy dispersion $w_{K}$. Where available, the Isothermal Remanent Magnetization curve (IRM) was simulated using the same parameters, thereby indicating the possible presence of biaxial corrections to the magnetic anisotropy [12]. An example set of curves is shown in figure 3. The obtained values are shown in table 1 .

\section{Discussion}

In table 1 a non-monotonous behavior of the magnetic anisotropy constant $K_{\text {eff }}$ is immediately visible, $K_{\text {eff }}$ it shows a minimum around $3.5 \mathrm{~nm}$ and increases from there for both decreasing and increasing sizes (cf. also fig. 4). A closer look at the magnetic anisotropy energy [14] allows identifying the contributions:

$$
M A E=K_{\text {eff }} V=E_{\text {shape }}+E_{\text {surface }}+E_{M E}+E_{M C} .
$$

Here we see the effect of the particle shape in the classical shape anisotropy energy $E_{\text {shape }}$ and of symmetry breaking at the surface in $E_{\text {surface }}$. Additional 
Table 1 Parameters obtained from the simultaneous fitting of the magnetic data and from the structural characterisation: the median magnetic diameter $D_{\text {mag }}$, its dispersion $w_{\text {mag }}$, the effective anisotropy constants $K_{\text {eff }}$ and its dispersion. Except for the sample with 4.7 $\mathrm{nm}$ diameter, where no IRM curve was measured, the ratio between the biaxial and the effective anisotropy constant $K_{2} / K_{\text {eff }}$ is obtained from simulations of the IRM curve at $2 \mathrm{~K}$. The error values were estimated in a thorough study of the confidence limits of the fit procedures $[10,13,12]$. Also shown are the mean aspect ratios $c / a$ (see text).

\begin{tabular}{cccccc}
$D_{m a g}(\mathrm{~nm})$ & $w_{m a g}$ & $K_{\text {eff }}\left(\mathrm{kJ} / \mathrm{m}^{3}\right)$ & $w_{K}$ & $K_{2} / K_{\text {eff }}$ & $c / a$ \\
\hline \hline $1.9 \pm 0.1$ & $0.10 \pm 0.01$ & $218 \pm 10$ & $0.40 \pm 0.1$ & $0 \pm 0.4$ & 1.16 \\
$2.7 \pm 0.1$ & $0.20 \pm 0.01$ & $135 \pm 10$ & $0.40 \pm 0.1$ & $0.8 \pm 0.4$ & 1.16 \\
$3.2 \pm 0.1$ & $0.17 \pm 0.01$ & $114 \pm 10$ & $0.35 \pm 0.1$ & $1.3 \pm 0.4$ & 1.2 \\
$3.5 \pm 0.1$ & $0.17 \pm 0.01$ & $110 \pm 10$ & $0.35 \pm 0.1$ & $1.1 \pm 0.4$ & 1.24 \\
$3.8 \pm 0.1$ & $0.18 \pm 0.01$ & $154 \pm 10$ & $0.40 \pm 0.1$ & $1.0 \pm 0.4$ & 1.3 \\
$4.7 \pm 0.25$ & $0.20 \pm 0.05$ & $182 \pm 15$ & $0.10 \pm 0.1$ & - & 1.46 \\
$5.5 \pm 0.1$ & $0.13 \pm 0.01$ & $170 \pm 10$ & $0.35 \pm 0.1$ & $1.2 \pm 0.4$ & 1.6 \\
\hline
\end{tabular}

terms take into account volume relaxation due to external stress (magnetoelastic anisotropy $E_{M E}$ ) and the cubic magnetocrystalline anisotropy $E_{M C}$. This expression can be expanded into a power series of the magnetization axes in space [15]. The shape anisotropy only contributes with a second order term, the surface and elastic anisotropy contributions start at second, the magnetocrystalline contribution at fourth order. As we have shown above our particles are crystallized in fcc structures. The bulk magnetocrystalline fcc anisotropy of $27 \mathrm{~kJ} / \mathrm{m}^{3}$ [16] is, however, much too low to explain our findings. We furthermore neglect the magnetoelastic term as the Young's modulus of cobalt of $209 \mathrm{GPa}$ is much bigger that that of copper (117 GPa), all stress can thus be relieved in the matrix rather than in the particle. This leaves shape and surface anisotropy as possibly responsible for the observed size effects and we now show how they can, respectively, explain the small and large size rise in $K_{\text {eff }}$.

We explain the rise in the effective anisotropy constant for the smallest particles using the phenomenological model introduced by Néel [17]. Here a pair interaction is described by one single parameter derived from magnetoelastic constants, therefore intimately connected to lattice variations due to relaxation as well as to symmetry breaking at the cluster surface. This model has been successfully used to evidence the primordial role of additional facets for the surface anisotropy [14]. We have calculated the effective anisotropy for model fcc structures showing the increasing importance of additional crystalline facets with decreasing size for particles with diameters below approximately $3 \mathrm{~nm}$. A closed-shell truncated octahedron has no additional surface anisotropy in this model but added facets contribute strongly. In figure 4 are shown the experimental values for $K_{e f f}$ in red as well as the calculated values for truncated octahedra with one additional facet along the [100] (blue) or the [111] direction (green), respectively. The calculated effective anisotropy constant increaes with decreasing size, in accordance with the experimental findings, even though an additional facet contains less atoms for smaller particle diameters. The microscopy data we present here support our argumen- 


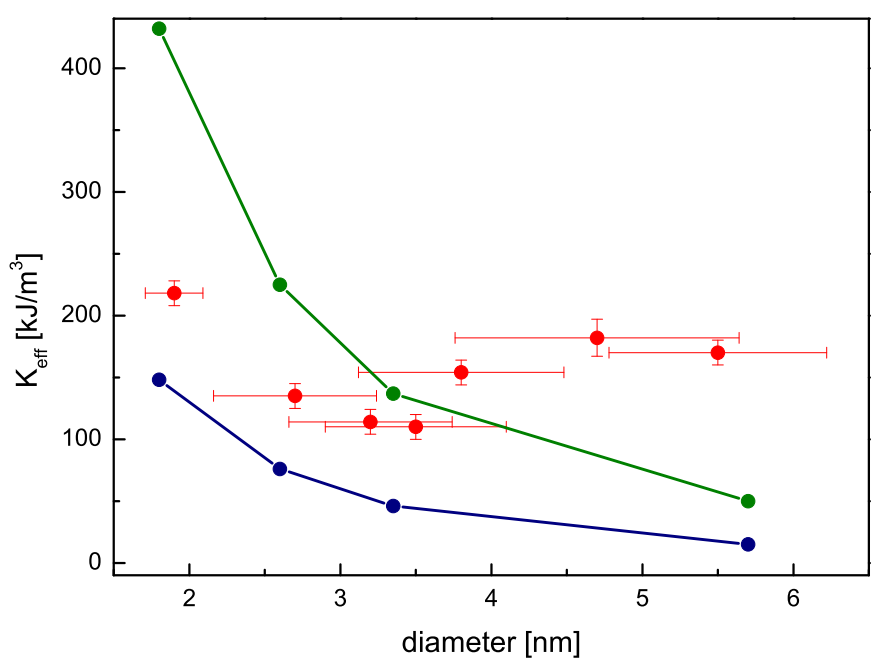

Fig. 4 (color online) Size dependency of the magnetic anisotropy constant. Values obtained from fits to experimental data are shown in red, simulated values for one additional facet along the [100] in blue or the [111] direction in green. The vertical bars are the errors derived from the fits, the horizontal bars correspond to the dispersions obtained for the magnetic cluster size.

tation by confirming the fcc structure used in the simulations. Note that the magnetocrystalline anisotropy of nanometer sized cobalt particles has been determined to $10 \mathrm{~kJ} / \mathrm{m}^{3}$ [16] and is thus too small to explain our observations. While we have not included the effect of twinning in our Néel model calculations, we estimate its impact to be minor, considering the decisive role of the surface observed also in the calculations performed for multiply twinned icosahedral structures [18]. Further improved calculations based on the Néel model are nevertheless scheduled for near future.

Larger particles, with diameters $>3 \mathrm{~nm}$, display deviations from a spherical shape due to the cluster fabrication process. At increased pressures in the aggregation tube particles grow not only by atom addition but also by cluster coalescence in the gas phase, the observed geometric anisotropy increases with particle size. We determined the aspect ratio c/a for a large number of particles by fitting ellipses to TEM images and find a mean value 1.16 for particle diameters below approximately $3 \mathrm{~nm}$, slightly bigger than unity due to facetting and the discrete pixel structure of the microscopy images. For larger particles we parametrize the aspect ratio as linearly increasing up to 1.6 for 6 nm diameter [4]. This aspect ratio can be then be converted to magnetic shape anisotropy [19]. The size dependent variations of the effective anisotropy constant $K_{\text {eff }}$ as a function of aspect ratio are displayed in figure 5 together with the calculated shape anisotropy. We find that the increasing ellipticity with 


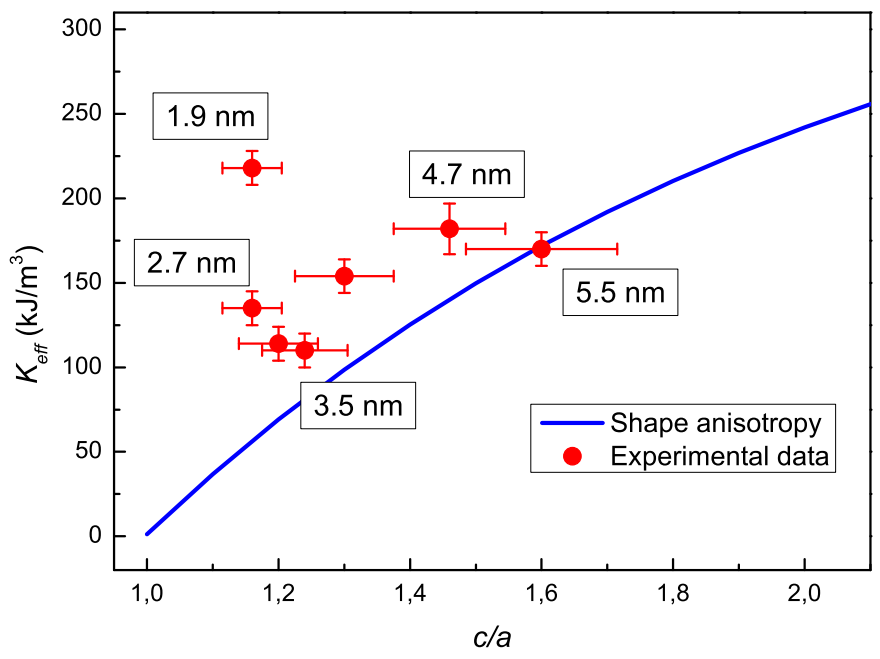

Fig. 5 (color online) Experimental variations of the magnetic anisotropy constant $K_{\text {eff } f \text { as }}$ a function of the aspect ratio $c / a$. The horizontal bars for the experimental data correspond to the dispersions of the aspect ratio as estimated from TEM. The blue line shows the calculated shape anisotropy constants.

increasing size can by itself explain the rise in effective magnetic anisotropy for larger sizes. The surface anisotropy is, however, not entirely negligible and leads to the observed difference between experiment and calculation. Note that the dispersions of the magnetic size distributions have been deliberately chosen as horizontal bars in order to represent the effective overlap between different samples.

The two models used thus reproduce the observed trends. For small clusters the nearly spherical shape means negligible shape anisotropy, the surface plays a dominant role. Larger particles show, at least with our fabrication technique, deviations from a sphere and thus a more and more important shape anisotropy with increasing size. The minimum in the curve in figure 4 is determined by the trade-off between the two contributions.

\section{Conclusions}

In this article we discuss size dependent variations of the magnetic anisotropy of cobalt nanoparticles embedded in copper matrices. We clearly show by HRTEM the fcc structure of the Co nanoparticles, thus allowing us to neglect the magnetocrystalline contribution to the anisotropy, as predicted in our previous paper [4]. With the new experimental data on the magnetic anisotropy the consistent picture is as follows: in small particles the contribution of sur- 
face anisotropy due to symmetry breaking caused by the addition of additional atoms at the interface is dominant, all the more the smaller the particle is. For sizes above $>\sim 3 \mathrm{~nm}$ diameter this contribution levels off and here deviations from a spherical shape lead to predominant shape anisotropy.

Acknowledgements We thank O. Boisron, C. Albin, C. Clavier and N. Blanchard for technical support. This work was performed using the Lyon Cluster Research Platform PLYRA and the Lyon Center for Magnetometry CML. This study was funded by the Science Without Borders "Special Visiting Scientist" program (grant number 88881.030488/201301) and through a "Chaire Française dans l'État de São Paulo" for M.H.. The authors declare that they have no conflict of interest.

Author contribution statement M.H. planned and supervised the research. S.O. and M.H. prepared the samples and performed the magnetic experiments, S.O., A.T., F.T. and M.H. treated the magnetic data. N.T. and J.R. performed the TEM experiments. M.H. wrote the manuscript. All authors participated in the discussion of the results and reviewed the manuscript.

\section{References}

1. R.L. Johnston, Atomic and Molecular Clusters (Taylor \& Francis, London, 2002)

2. U. Kreibig, M. Vollmer, Optical properties of metal clusters. Springer series in materials science (Springer Berlin, 1995)

3. J.M.D. Coey, Magnetism and Magnetic Materials (Cambridge University Press, 2010)

4. S. Oyarzún, A. Tamion, F. Tournus, V. Dupuis, M. Hillenkamp, Sci. Rep. 5, 14749 (2015). DOI 10.1038/srep14749

5. M. Hillenkamp, G. Di Domenicantonio, C. Félix, Rev. Sci. Instrum. 77, 025104 (2006). DOI 10.1063/1.2173051

6. M. Hillenkamp, G. Di Domenicantonio, C. Félix, Phys. Rev. B 77, 014422 (2008)

7. S. Oyarzún, A. Domingues Tavares de Sa, J. Tuaillon-Combes, A. Tamion, A. Hillion, O. Boisron, A. Mosset, M. Pellarin, V. Dupuis, M. Hillenkamp, J. Nanopart. Res. 15, 1968 (2013). DOI 10.1007/s11051-013-1968-8

8. A. Tamion, C. Raufast, M. Hillenkamp, E. Bonet, J. Jouanguy, B. Canut, E. Bernstein, O. Boisron, W. Wernsdorfer, V. Dupuis, Phys. Rev. B 81, 144403 (2010). DOI 10.1103/PhysRevB.81.144403

9. A. Tamion, M. Hillenkamp, A. Hillion, F. Tournus, J. Tuaillon-Combes, O. Boisron, S. Zafeiratos, V. Dupuis, J. Appl. Phys. 110, 063904 (2011). DOI 10.1063/1.3638035

10. A. Tamion, M. Hillenkamp, F. Tournus, E. Bonet, V. Dupuis, Appl. Phys. Lett. 95, $062503(2009)$

11. F. Tournus, N. Blanc, A. Tamion, M. Hillenkamp, V. Dupuis, Phys. Rev. B 81, 220405 (2010). DOI 10.1103/PhysRevB.81.220405

12. A. Hillion, A. Tamion, F. Tournus, O. Gaier, E. Bonet, C. Albin, V. Dupuis, Phys. Rev. B 88, 094419 (2013)

13. A. Tamion, E. Bonet, F. Tournus, C. Raufast, A. Hillion, O. Gaier, V. Dupuis, Phys. Rev. B 85, 134430 (2012). DOI 10.1103/PhysRevB.85.134430

14. M. Jamet, W. Wernsdorfer, C. Thirion, D. Mailly, V. Dupuis, P. Mélinon, A. Pérez, Phys. Rev. Lett. 86, 4676 (2001). DOI 10.1103/PhysRevLett.86.4676

15. M. Jamet, W. Wernsdorfer, C. Thirion, V. Dupuis, P. Mélinon, A. Pérez, D. Mailly, Phys. Rev. B 69, 024401 (2004)

16. J.P. Chen, C.M. Sorensen, K.J. Klabunde, G.C. Hadjipanayis, J. Appl. Phys. 76(10), 6316 (1994). DOI http://dx.doi.org/10.1063/1.358280

17. L. Néel, J. Phys. Radium 15, 376 (1954). DOI 10.1051/jphysrad:01954001505037601

18. R. Morel, A. Brenac, C. Portemont, T. Deutsch, L. Notin, J. Magn. Magn. Mater. 308 296 (2007). DOI http://dx.doi.org/10.1016/j.jmmm.2006.06.004

19. J.A. Osborn, Phys. Rev. 67, 351 (1945). DOI 10.1103/PhysRev.67.351 